VOL. 51 (1995) [133-137]

\title{
A NOTE ON OHM'S RATIONALITY CRITERION FOR CONICS
}

\author{
Mowaffaq HajJa
}

\begin{abstract}
An irreducible quadratic polynomial $P(X, Y)$ in two variables over a field $k$ is called a conic over $k$. It is called rational if its function field is simple transcendental over $k$ (equivalently if $P$ is parameterisable by rational functions). Ohm's rationality criterion states that $P$ is rational if and only if (i) the locus of $P$ is non-empty and (ii) $k$ is algebraically closed in the function field of $P$. To show the irredundancy of (ii), Ohm gives an example of a non-rational conic with a non-empty locus. That locus, however, consists of a single point.

In this note, we show that a better example cannot exist by showing that if the locus of a conic contains more than one point then it is rational. We also show that the only rational conic whose locus consists of one point is the conic $X Y+1$ over the field of two elements.
\end{abstract}

Let $k$ be any field. An irreducible polynomial $P=P(X, Y) \in k[X, Y]$ of total degree 2 is called a conic over $k$ (or simply a conic). A point $(\alpha, \beta) \in k^{2}$ with $P(\alpha, \beta)=$ 0 is called a $k$-zero of $P$ and the set of all $k$-zeros of $P$ is referred to as the locus $P$. The quotient field of $k[X, Y] /(P)$ is called the function field of $P$. If one denotes the images of $X, Y$ under the canonical map

$$
k[X, Y] \rightarrow k[X, Y] /(P)
$$

by $x, y$, then the function field of $P$ is nothing but the field extension $k(x, y)$ of $k$. It is easy to see that the generators $x, y$ are characterised by the properties that (i) the transcendence degree $d t_{k} k(x, y)$ of $k(x, y)$ over $k$ is 1 and (ii) the ideal of polynomials in $k[X, Y]$ that vanish on $(x, y)$ is the principal ideal generated by $P$. Also, it is easy to see that a non-singular affine change of variables

$$
X \rightarrow a X+b Y+c, \quad Y \rightarrow \alpha X+\beta Y+\gamma
$$

transforms $P$ into a conic $Q$ having a $k$-isomorphic function field. Such conics $P, Q$ are called equivalent.

The function field $k(x, y)$ of $P$ is said to be rational if it is simple transcendental over $k$, that is if it is generated by a single element $t$. In this case, $P$ itself is called

Received 11 April 1994

Copyright Clearance Centre, Inc. Serial-fee code: 0004-9729/95 SA2.00+0.00. 
a rational conic. It is easy to see that the rationality of a conic is equivalent to its parameterisability by rational functions (in the variable $t$ ).

In [1, Theorem 1.4, (ii) $\Longleftrightarrow$ (iii)] Ohm gives necessary and sufficient conditions for a conic to be rational. His criterion asserts that the function field $k(x, y)$ of the conic $P$ is rational if and only if the following two conditions are satisfied:

(A) $P$ has a $k$-zero (that is, $P$ has a non-empty locus)

(B) $k$ is algebraically closed in $k(x, y)$.

At the end of the proof, he remarks that $(B)$ is not redundant by considering the function field of the conic $P(X, Y)=X^{2}+Y^{2}$ over the field of real numbers.

The example above does indeed satisfy (A) (since $P(0,0)=0$ ) and does not satisfy (B) (since $y / x$ is an element in $k(x, y)-k$ that is algebraic over $k$ ). However, it satisfies (A) only very weakly: true, the locus of $P$ is not empty, but it is almost empty since it consists of the single point $(0,0)$. This observation leads naturally to the search for a non-rational conic whose locus contains more than one point.

In this note, we prove that if the locus of a conic $P$ has more than one point, then $P$ is rational. Further, we prove that the only rational conic whose locus is a singleton is the conic

$$
P(X, Y)=X Y+1, \quad k=\mathbb{Z}_{2} .
$$

THEOREM 1. Let $k(x, y)$ be the function field of the conic $P$ and suppose that the locus of $P$ is nonempty. If $P$ is not rational then the locus of $P$ consists of a single point. In this case, $P$ is equivalent to

$$
a X^{2}+b X Y+c Y^{2} .
$$

Conversely, if $P$ is irreducible of the form above, then the locus of $P$ consists of a single point and $P$ is not rational.

Proof: Since the locus of $P$ is not empty, then by a suitable change of variables, one may assume that $(0,0)$ lies on the locus of $P$. Thus $P(0,0)=0$ and hence

$$
P(X, Y)=a X^{2}+b X Y+c Y^{2}+d X+e Y .
$$

Suppose that $P$ is not rational. If $x=0$, then $c y^{2}+e y=0$ and hence $y \in k$. Hence $k(x, y)=k$, contradicting the assumption that $d t_{k} k(x, y)=1$. Hence $x \neq 0$. Let $t=y / x$. Then

$$
\left(a+b t+c t^{2}\right) x+(d+d t)=0 .
$$

If $\left(a+b t+c t^{2}\right) \neq 0$, then $x$ would belong to $k(t)$. Hence

$$
k(x, y)=k(x, x t)=k(t)
$$


contradicting the assumption that $k(x, y)$ is not rational. Thus $a+b t+c t^{2}=0$ and therefore $d+e t=0$. But $t \notin k$ (since $k(x, y) \neq k(x)$ ). So then $e=d=0$. Therefore

$$
P(X, Y)=a X^{2}+b X Y+c Y^{2} .
$$

Let $(r, s)$ be another $k$-zero of $P$. Since $P$ is irreducible, then neither $a$ nor $c$ is 0 . Therefore neither $r$ nor $s$ is 0 . Hence

$$
s X-r Y
$$

is a factor of $P$, contradicting the irreducibility of $P$. This shows that $(0,0)$ is the only $k$-zero of $P$ (and that $P$ is equivalent to the given form).

Conversely, if $P$ is irreducible and of the given form, then the same argument above shows that the locus of $P$ contains no point other than $(0,0)$ and that $y / s$ is an element in $k(x, y)-k$ that is algebraic over $k$. Thus $k$ is not algebraically closed in $k(x, y)$ and hence $P$ is not rational.

COROLlary 2. If the locus of the conic $P$ has more than one point, then $P$ is rational.

Can the locus of a rational conic consist of a single point? The following example shows the existence of such a conic, while the theorem that follows shows its uniqueness.

Example 3. Let $k=\mathbb{Z}_{2}$ and let $P(X, Y)=X Y+1$. To see that the locus of $P$ has exactly one point, we try all possibilities

$$
(0,0),(0,1),(1,0),(1,1)
$$

and easily see that $(1,1)$ is the only zero of $P$. To see that it is rational, we note that $y=1 / x$ and hence $k(x, y)=k(x)$.

TheOREM 4. If the locus of the $k$-conic $P$ consists of a single point, and if $P$ is rational then $k=\mathbb{Z}_{2}$ and $P$ is equivalent to $X Y+1$.

Proof: Let $K=k(x, y)$ be the function field of $P$ (with $P(x, y)=0$ ). Suppose that $P$ is rational and that the locus of $P$ consists of a single point. Then by a suitable change of variables, one may assume that point to be $(0,0)$. Then

$$
P(X, Y)=a X^{2}+b X Y+c Y^{2}+d X+e Y .
$$

If $x=0$, then $c y^{2}+e y=0$ and hence $y \in k$. This contradicts the fact that $d t_{k} k(x, y)=$ 1 . Therefore $x \neq 0$. Let $t=y / x$. Then

$$
x\left[a+b t+c t^{2}\right]+[d+e t]=0 .
$$


If $a+b t+c t^{2}=0$, then

$$
a x^{2}+b x y+c y^{2}=0
$$

and hence

$$
P(X, Y)=a X^{2}+b X Y+c Y^{2} .
$$

In view of Theorem 1, this contradicts the rationality of $P$. Therefore

$$
a+b t+c t^{2} \neq 0
$$

and hence $d+e t \neq 0$. Hence $(a, b, c) \neq(0,0,0)$ and

$$
(d, e) \neq(0,0) \text {. }
$$

Also,

$$
x=-[d+e t] /\left[a+b t+c t^{2}\right], \quad y=-t[d+e t] /\left[a+b t+c t^{2}\right] .
$$

If $k$ has more than 3 elements, then one can find $\alpha$ in $k$ such that

$$
(d+e \alpha)\left(a+b \alpha+c \alpha^{2}\right) \neq 0 .
$$

Then

$$
\left(-(d+e \alpha) /\left(a+b \alpha+c \alpha^{2}\right),-\alpha(d+e \alpha) /\left(a+b \alpha+c \alpha^{2}\right)\right)
$$

would be another point on the locus of $P$, contrary to the hypothesis. Thus $k$ cannot have more than 3 elements and consequently $k$ must be either $\mathbb{Z}_{2}$ or $\mathbb{Z}_{3}$.

CASE 1. $k=\mathbb{Z}_{2}$. Plugging $Y=0$ in $(*)$, we see that if $d a \neq 0$ then $(-d / a, 0)$ is another $k$-zero of $P$, contrary to the hypothesis. Therefore $d a=0$. Similarly $e c=0$. Also plugging $X=Y$ in $(*)$, we see that if $(a+b+c)(d+e) \neq 0$, then $((d+e) /(a+b+c),(d+e) /(a+b+c))$ is another $k$-zero of $P$. Hence we conclude that

$$
d a=e c=(d+e)(a+b+c)=0 .
$$

If $(d, e)=(1,1)$, then $a=c=0$ and

$$
P(X, Y)=X Y+X+Y=(X+1)(Y+1)+1 .
$$

Making the change of variables

$$
X \rightarrow X+1, \quad Y \rightarrow Y+1
$$

we see that $P$ is equivalent to $X Y+1$. 


$$
\begin{aligned}
& \text { If }(d, e)=(1,0), \text { then } a=b+c=0 . \text { Hence } b=c \text { and } \\
& \qquad P(X, Y)=X Y+Y^{2}+X=(X+Y+1)(Y+1)+1 .
\end{aligned}
$$

Making the change of variables

$$
X \rightarrow X+Y+1, \quad Y \rightarrow Y+1
$$

we see that $P$ is equivalent to $X Y+1$.

The case $(d, e)=(0,1)$ is similar while the case $(d, e)=(0,0)$ is not feasible by (1).

Hence in all cases, $P$ is equivalent to $X Y+1$ as desired.

CASE 2: $k=\mathbb{Z}_{3}$. Plugging $Y=0$ in $(*)$, we see that if $d a \neq 0$ then $(-d / a, 0)$ is another $k$-zero of $P$, contrary to the hypothesis. Therefore $d a=0$. Similarly by plugging $X=0, X=Y, X=-Y$, we conclude that

$$
d a=e c=(d+e)(a+b+c)=(d-e)(a-b+c)=0
$$

Case by case computations reveal that every case leads to a contradiction.

Thus the only rational conic whose locus consists of a single point is the conic $X Y+1$ over $\mathbb{Z}_{2}$.

In conclusion, we summarise our results for a conic $P$ over a field $k$ as follows:

(i) If the locus of $P$ is empty, then $P$ is not rational.

(ii) If the locus of $P$ contains more than one point, then $P$ is rational.

(iii) If the locus of $P$ consists of one point, then (a) $P$ is not rational if and only if $P$ is equivalent to an irreducible polynomial of the form $a X^{2}+$ $b X Y+c Y^{2}$ and (b) $P$ is rational if and only if $k=\mathbb{Z}_{2}$ and $P$ is equivalent to $X Y+1$.

\title{
REFERENCES
}

[1] J. Ohm, 'Function fields of conics, a theorem of Amitsur-MacRae, and a problem of Zariski', in Algebraic Geometry and its applications, (C. Bajaj, Editor) (Springer-Verlag, Berlin, Heidelberg, New York, 1993), pp. 351-382.

\author{
Mathematics Department \\ Yarmouk University \\ Irbid \\ Jordan
}

